\title{
Monoamniotic twins discordant for body stalk anomaly
}

\author{
Mariana Vide Tavares, Ana Patrícia Domingues, Margarida Tavares, Etelvina Fonseca, and Paulo Moura \\ Obstetric Department A, Centro Hospitalar e Universitário de Coimbra, Coimbra, Portugal
}

\begin{abstract}
Body stalk anomaly is a rare malformation. This anomaly in monozygotic twins is extremely unusual. We describe a case of monoamniotic pregnancy discordant for body stalk anomaly diagnosed at 11 weeks. Ultrasound showed a fetus with a large anterior abdominal wall defect, anomaly of the spine and no evidence of lower extremities and other with a normal morphology. As far as our concern, only three monoamniotic pregnancies discordant for this malformation were reported. Our case represents the fourth reported monoamniotic pregnancy discordant for body stalk anomaly with diagnosis made by ultrasound and the second diagnosed in the first trimester.
\end{abstract}

\author{
Keywords \\ Body stalk anomaly, monoamniotic

\section{History} \\ Received 4 February 2013 \\ Accepted 27 February 2014 \\ Published online 9 April 2014
} pregnancy, prenatal diagnosis, ultrasound

\section{Introduction}

Body stalk anomaly is a rare and lethal condition. The prevalence is 1 in 7500 pregnancies between 10 and 14 weeks [1]. The presence of this anomaly in monozygotic twins is extremely unusual [2]. As far as our concern, only five cases of monozygotic twins discordant for this malformations were reported [2-5]; three in monoamniotic pregnancies [2-4]. Our case represents the fourth [2,3,5] reported monoamniotic pregnancy discordant for body stalk anomaly with diagnosis made by ultrasound and the second [2] diagnosed in the first trimester.

\section{Case report}

A healthy 37-year-old primigravid woman with 11 weeks of gestation was referred to our center for an abnormal fetus in a twin pregnancy. The sonographic examination revealed a monoamniotic twin pregnancy with a normal fetus (fetus A) and other severely malformed (fetus B). Fetus A had a normal morphology and a normal nuchal translucency thickness for the crown-rump length (CRL) (Figure 1). Fetus B had a large anterior abdominal wall defect, anomaly of the spine and no evidence of lower extremities (Figure 2). It seems to be attached to the placenta at the abdominal level (Figure 2). Nuchal translucency thickness was above the 95th percentile for CRL (Figure 2). The umbilical cord of fetus B was shorter than fetus A (Figure 3). The diagnosis of body stalk anomaly was suspected. Parents were counseled as to the condition of abnormal fetus and they chose to undergo a termination of pregnancy with prostaglandin induction. The fetal autopsy showed that fetus A had no abnormalities. Fetus B had an

Address for correspondence: Mariana Vide Tavares, Obstetric Department A, Centro Hospitalar e Universitário de Coimbra, Rua Miguel Torga, 3030-165 Coimbra, Portugal. Tel: +351917050588, +351224092738. E-mail: m.vide@hotmail.com eventration of abdominal organs and a deformed thoracic cavity, absence of one lower limb and the other deformed and attached to the dorsal region. A single placenta with an intact single membrane was identified. Two umbilical cords with two arteries and one vessel were observed; the one of fetus B was twice fold shorter and attached to the internal surface of the placenta. The diagnosis of body stalk anomaly was confirmed. No karyotype examination was performed by insufficient material.

\section{Discussion}

The diagnosis of body stalk anomaly can be made by ultrasound assessment at 11-14 weeks [1] and can identify cases as earlier as the beginning of the 11 weeks, as our case illustrates.

Body stalk anomaly is characterized by the presence of major anterior body wall defect, abnormal limb deformities, kyphoscoliosis, an absent or short umbilical cord and/or craniofacial defects. It is not associated with chromosomal abnormalities [4].

Differential diagnosis has to be made with other fetal abdominal wall defects such as amniotic band syndrome, gastroschisis, omphalocele, Pentalogy of Cantrell and others [4].

Different pathogenic theories could explain this anomaly: early amnion rupture, vascular disruption and embryonic maldevelopment. Early amnion rupture before the obliteration of the celomic cavity will lead to the formation of fibrous bands from the exposed chorionic surface and cause traumatic lesions on fetal body [5] and/or contribute for a part of the body to be in the exocelomic cavity [1]. No amniotic rupture was observed in our case unlike other reports [1,2]. The intact membrane has been confirmed by pathologic examination. Therefore, our case challenges the validity of the theory of early amniotic rupture. Beside the intact amniotic membrane, 


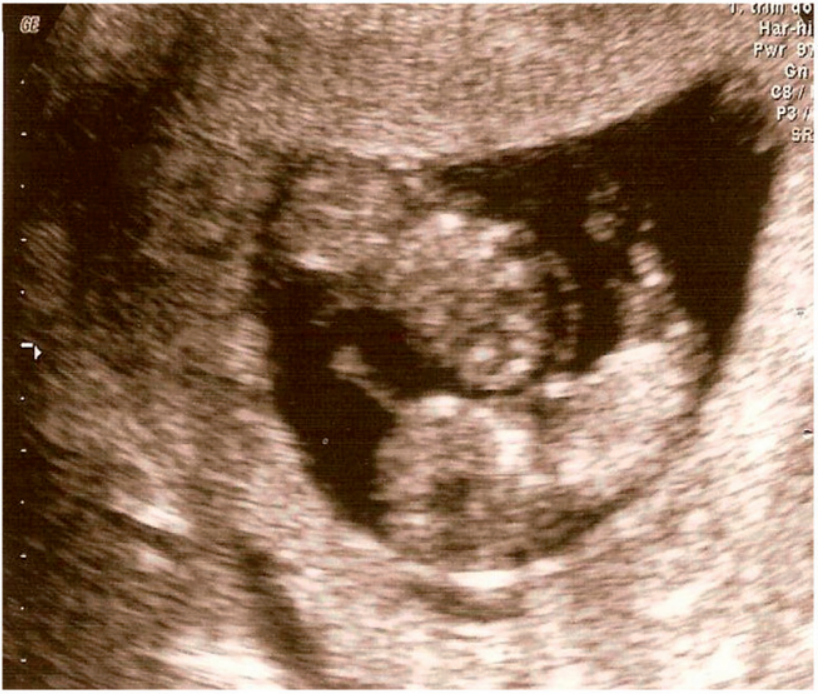

Figure 1. Normal fetus with a normal morphology in the bottom of the figure. In the upper part, the abnormal fetus. No evidence of amniotic membrane.

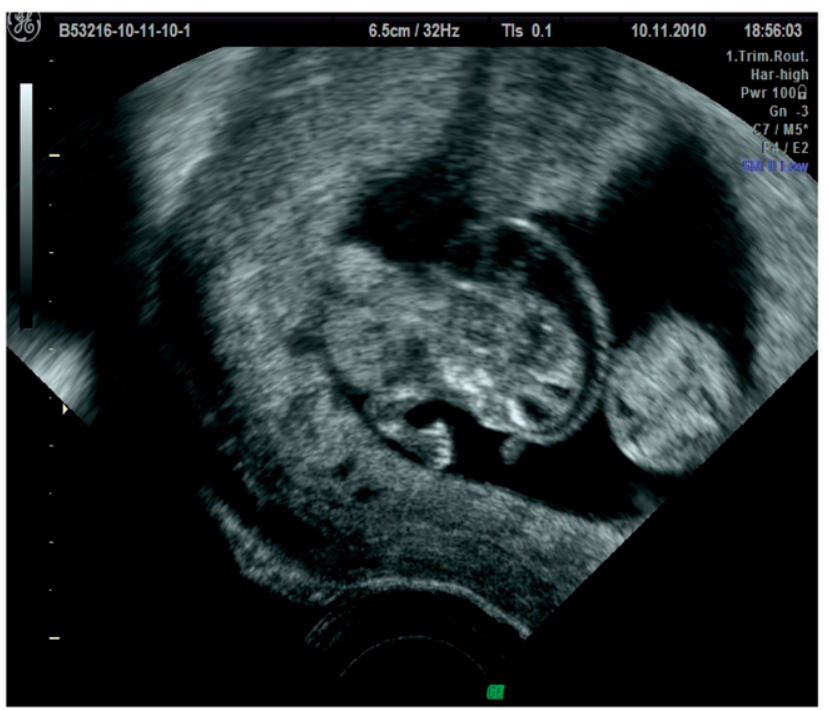

Figure 2. Nuchal translucency clearly increased in abnormal fetus. Biparietal diameter (BPD) section of normal fetus. No evidence of amniotic membrane.

in our case, the abnormal fetus was attached to the placenta and had a short umbilical cord also adherent to placenta. So, the hypothesis proposed by Daskalakis et al. [1] cannot be confirmed. The marked shortening of the umbilical cord can contribute, by itself, to the abdominoplacental attachment [5]. A case reported by Paul et al. [6] as well as ours is against this theory and shows that it is not a necessary event in the development of body stalk anomaly [5].

Van Allen et al. [7] suggested that early compromise of embryonic blood flow during the first 4-6 weeks, leads to failure of closure of the ventral body wall and persistence of the extra-embryonic celomic cavity. Others studies showed an association of body stalk anomaly and maternal cocaine abuse. In our case, this theory found no evidence because there was no history of drug abuse or vasoconstrictive medication.

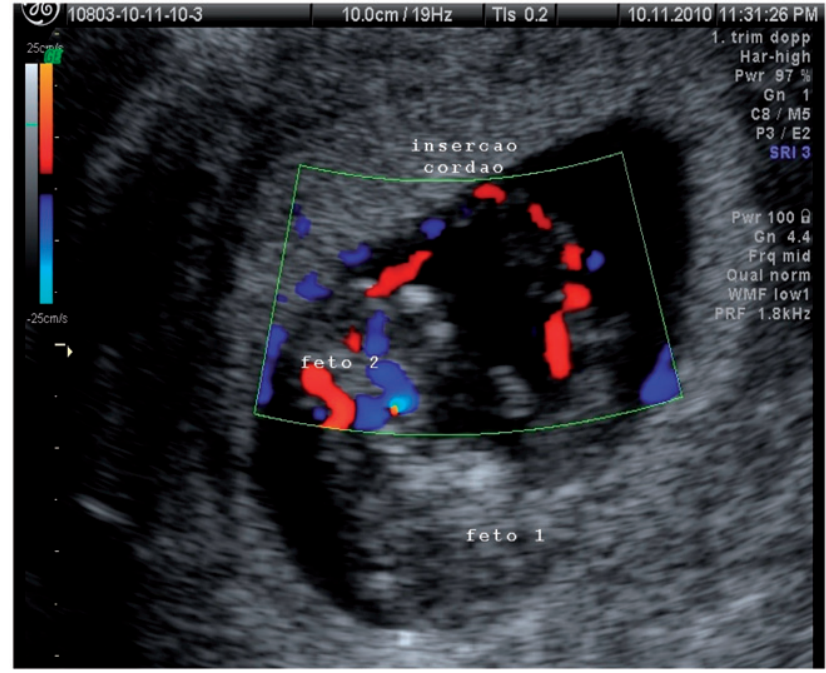

Figure 3. Vascular relations between the two fetuses. The length of the umbilical cord of abnormal fetus (fetus 2) is shorter than in the normal one.

Vidaeff et al. [5] also suggested that these two theories are difficult to reconcile with the occurrence of discordant body stalk anomaly in monoamniotic pregnancies. The generalized nature of the changes proposed cannot explain the discordant occurrence of this anomaly and the undisturbed evolution of the unaffected fetus as illustrated by Daskalakis and Nicolaides [2].

Embryonic maldevelopment is the third and most accepted theory and consists in a defect in the germinal disk. During 5th week of gestation, the flat trilaminar embryo is transformed into a cylindrical fetus by a parallel set of four contiguous body folds (cephalic, caudal and lateral folds). Body stalk anomaly is supposed to be due to faulty in all three axes with persistence of the extra-embryonic celomic cavity and the various malformations depends on the degree of aberrant development of each folds [4].

Expectant management was chosen in the other cases of monoamniotic twin discordant for body stalk anomaly reported in literature. An elective C-section at 35 weeks of gestation was performed by Daskalakis and Nicolaides [2] and in the case reported by Vidaeff et al. [5], a spontaneous vaginal delivery occurred at 34 weeks of gestation. In our case, the parents had opted for the termination of pregnancy.

It is not only important the survival but also the neonatal outcome. When the expectant management is chosen, the outcome of the unaffected fetus should be anticipated [8]. The risk of perinatal morbidity and mortality after spontaneous or iatrogenic pre-term delivery of the unaffected twin must be weighed as well as the possible risks to the surviving twin in the case of intrauterine death of the malformed fetus.

In conclusion, the body stalk anomaly can be diagnosed as earlier as the 11 weeks of gestation. The differential diagnosis with other conditions should be made. We believe that embryonic maldevelopment could explain the body stalk anomaly, however, without excluding the other conditions contribution. The management should be individualized. Parents have the last call. The limited number of cases does not permit us to obtain consistent data from the perinatal outcome of the unaffected twin. 


\section{Declaration of interest}

The authors report no declarations of interest.

\section{References}

1. Daskalakis G, Sebire NJ, Jurkovic D, et al. Body stalk anomaly at 10-14 weeks of gestation. Ultrasound Obstet Gynecol 1997;10: 416-18.

2. Daskalakis GJ, Nicolaides KH. Monozygotic twins discordant for body stalk anomaly. Ultrasound Obstet Gynecol 2002;20:79-81.

3. Khudr G, Benirscke K. Discordant monozygous twins associated with amnion rupture: a case report. Obstet Gynecol 1972;39: 713-16.
4. Smreck JM, Germer U, Krokowwski M, et al. Prenatal ultrasound diagnosis and management of body stalk anomaly: analysis of nine singleton and two multiple pregnancies. Ultrasound Obstet Gynecol 2003;21:322-8.

5. Vidaeff AC, Delu AN, Silva JB, Yeomans ER. Monoamniotic twin pregnancy discordant for body stalk anomaly. J Ultrasound Med 2005;24:1739-44.

6. Paul C, Zosmer N, Jurkovic D, Nicolaides K. A case of body stalk anomaly at 10 weeks of gestation. Ultrasound Obstet Gynecol 2001; 17:157-9.

7. Van Allen M, Curry C, Gallagher L. Limb-body wall complex: I. Pathogenesis. Am J Med Genet 1987;28:529-48.

8. Kähler C, Hmbsch K, Schneider U, Seewald HJ. A case report of body stalk anomaly complicating a twin pregnancy. Arch Gynecol Obstet 2003;268:245-7. 